\title{
A multiplicity result for Chern-Simons- Schrödinger equation with a general nonlinearity
}

Patricia L. Cunha, Pietro d'Avenia, Alessio Pomponio and Gaetano Siciliano

Abstract. In this paper we give a multiplicity result for the following Chern-Simons-Schrödinger equation

$$
-\Delta u+2 q u \int_{|x|}^{\infty} \frac{u^{2}(s)}{s} h_{u}(s) d s+q u \frac{h_{u}^{2}(|x|)}{|x|^{2}}=g(u), \quad \text { in } \mathbb{R}^{2},
$$

where $h_{u}(s)=\int_{0}^{s} \tau u^{2}(\tau) d \tau$, under very general assumptions on the nonlinearity $g$. In particular, for every $n \in \mathbb{N}$, we prove the existence of (at least) $n$ distinct solutions, for every $q \in\left(0, q_{n}\right)$, for a suitable $q_{n}$.

Mathematics Subject Classification. 35J20, 35Q55, 81T10.

Keywords. Chern-Simons gauge field, Schrödinger equation, Variational methods, Radial solutions, General nonlinearities.

\section{Introduction}

This paper is devoted to the study of the following Chern-Simons-Schrödinger equation

$$
-\Delta u+2 q u \int_{|x|}^{\infty} \frac{u^{2}(s)}{s} h_{u}(s) d s+q u \frac{h_{u}^{2}(|x|)}{|x|^{2}}=g(u) \text { in } \mathbb{R}^{2},
$$

where $u: \mathbb{R}^{2} \rightarrow \mathbb{R}, q>0$ and $h_{u}(s)=\int_{0}^{s} \tau u^{2}(\tau) d \tau$. This equation describes nonrelativistic matter interacting with Chern-Simons gauge fields in the plane, under a suitable ansatz. For the reader convenience, in Sect. 2, we will give the physical motivations of this model, the derivation and justification of (1.1).

P. d'Avenia and A. Pomponio are supported by GNAMPA project "Aspetti differenziali e geometrici nello studio di problemi ellittici quasilineari". G. Siciliano is supported by Fapesp (SP) and CNPq, Brazil. 
Arguing as in [6, Proposition 2.2], solutions of (1.1) are critical points of the $C^{1}$-functional

$$
J_{q}(u)=\frac{1}{2} \int_{\mathbb{R}^{2}}|\nabla u|^{2} d x+\frac{q}{2} \int_{\mathbb{R}^{2}} \frac{u^{2}(|x|)}{|x|^{2}}\left(\int_{0}^{|x|} s u^{2}(s) d s\right)^{2} d x-\int_{\mathbb{R}^{2}} G(u) d x
$$

defined on $H_{r}^{1}\left(\mathbb{R}^{2}\right)=\left\{u \in H^{1}\left(\mathbb{R}^{2}\right): u\right.$ is radially symmetric $\}$, where $G(s)=$ $\int_{0}^{s} g(\tau) d \tau$. As we can see, the functional includes a nonlocal term which requires a careful analysis.

Our aim is to study (1.1) with a Berestycki, Gallouët and Kavian type nonlinearity [2], which satisfies very general and almost necessary conditions, and also it is the planar version of the Berestyski-Lions type nonlinearity $[3,4]$. These papers concern with the existence of nontrivial radial solutions for the following autonomous nonlinear field equation

$$
\left\{\begin{array}{l}
-\Delta u=g(u) \text { in } \mathbb{R}^{N}, \\
u \in H^{1}\left(\mathbb{R}^{N}\right),
\end{array}\right.
$$

with $N \geqslant 2$, by assuming

(g0) $g \in C(\mathbb{R}, \mathbb{R})$ is an odd function.

(g1) For $N \geqslant 3$,

$$
\limsup _{\xi \rightarrow \infty} \frac{g(\xi)}{\xi^{\frac{N+2}{N-2}}} \leqslant 0
$$

For $N=2$,

$$
\limsup _{\xi \rightarrow \infty} \frac{g(\xi)}{e^{\alpha \xi^{2}}} \leqslant 0, \quad \forall \alpha>0
$$

(g2) For $N \geqslant 3$,

$$
-\infty<\liminf _{\xi \rightarrow 0} \frac{g(\xi)}{\xi} \leqslant \limsup _{\xi \rightarrow 0} \frac{g(\xi)}{\xi}<0 .
$$

For $N=2$,

$$
-\infty<\lim _{\xi \rightarrow 0} \frac{g(\xi)}{\xi}<0 .
$$

(g3) There exists $\zeta_{0}>0$ such that $G\left(\zeta_{0}\right)>0$.

As we can see, there is a difference in the assumption (g2) between the cases $N \geqslant 3$ and $N=2$. The existence of a $\operatorname{limit}_{\lim _{\xi \rightarrow 0}} g(\xi) / \xi \in$ $(-\infty, 0)$, when $N=2$, is essential in the arguments of Berestycki et al. [2] to prove the Palais-Smale [(PS) from now on] condition for the corresponding functional under suitable constraint.

Later on, in a more recent paper, Hirata et al. [10] consider Eq. (1.3) in the case $N \geqslant 2$ assuming (g0), (g1), (g3) and

$\left(\mathrm{g} 2^{\prime}\right)-\infty<\liminf _{\xi \rightarrow 0} \frac{g(\xi)}{\xi} \leqslant \limsup _{\xi \rightarrow 0} \frac{g(\xi)}{\xi}<0$ 
and find radial solutions as critical points of the functional

$$
I(u)=\frac{1}{2} \int_{\mathbb{R}^{N}}|\nabla u|^{2} d x-\int_{\mathbb{R}^{N}} G(u) d x .
$$

Following the approach introduced by Jeanjean in [14], Hirata et al. [10] consider the auxiliary functional $\tilde{I}: \mathbb{R} \times H_{r}^{1}\left(\mathbb{R}^{N}\right) \rightarrow \mathbb{R}$

$$
\tilde{I}(\theta, u)=\frac{e^{(N-2) \theta}}{2} \int_{\mathbb{R}^{N}}|\nabla u|^{2} d x-e^{N \theta} \int_{\mathbb{R}^{N}} G(u) d x .
$$

In this way they are able to find a (PS) sequence $\left(\theta_{j}, u_{j}\right)$ such that $\theta_{j} \rightarrow 0$ and $u_{j}$ "almost" satisfies the Pohožaev identity associated to (1.3). Using this extra information, it is proved that problem (1.3) possesses a positive least energy solution and infinitely many (possibly sign changing) radially symmetric solutions.

We prove

Theorem 1.1. Assume (g0), (g1), (g2') and (g3). For every $n \in \mathbb{N}$ there exists $q_{n}>0$ such that, for every $q \in\left(0, q_{n}\right), E q$. (1.1) admits (at least) $n$ distinct solutions.

Of course the solutions will appear in pairs, since the functional is even. Moreover as in [6, Proposition 2.2] it can be seen that the solutions are classical.

Due to the presence of the nonlocal term, the method in [10] seems to be not sufficient and so we have to combine it with a penalization technique introduced in $[5,15]$. A similar approach has been used in [1] to the study a perturbed version of (1.3), in the case $N \geqslant 3$, introducing a family of auxiliary functionals suitably related to the "original" one. In our case, we consider the same family of functionals but we cannot repeat the same arguments.

One of the main difficulties and one of the main differences regarding the previous works is the proof of a uniform boundedness in $H_{r}^{1}\left(\mathbb{R}^{2}\right)$ of a suitable (PS) sequence $\left(u_{j}\right)_{j}$ with respect to $q$, at least for small positive $q$. In [1] the key point is to show a suitable bound of $\left(u_{j}\right)_{j}$ in $D^{1,2}\left(\mathbb{R}^{N}\right)$, and then the boundedness of the same sequence in $L^{2}\left(\mathbb{R}^{N}\right)$ follows by the continuous embedding of $D^{1,2}\left(\mathbb{R}^{N}\right)$ into $L^{2^{*}}\left(\mathbb{R}^{N}\right)$, where $N \geqslant 3$ and $2^{*}=2 N /(N-$ $2)$. In two dimensional case, however, these arguments do not work. We get a such uniform boundedness using an appropriate diagonalization argument. Moreover, while in [1], the hypothesis (g2) guarantees suitable compactness properties, in our case the assumption (g2') creates several problems with the lack of compactness. This is also the reason why our problem does not fall even into the case considered in [16].

We recall now some recent results concerning the equation under study. The case $g(u)=|u|^{p-1} u-\omega u$, where $p>1$ and $\omega>0$ is the phase of the standing wave $\psi(t, x)=u(x) e^{i \omega t}$, has been considered by several authors showing how the existence of solutions and the geometrical aspect of the EulerLagrange functional associated to (1.1) depend strongly on $p$ and $\omega$. In Byeon et al. [6], show that, for $p \geq 3$, the Euler-Lagrange functional is unbounded from below and exhibits a mountain-pass geometry for $p>3$. However the 
existence of a solution is not so direct, since for $p \in(3,5)$ the (PS) condition is not known to hold. This problem is bypassed by using a constrained minimization taking into account the Nehari and Pohožaev identities. In the special case $p=3$, with a suitable choice of physical constants, they prove that solutions can be found by passing to a self-dual equation, which leads to a Liouville equation that can be solved explicitly. Those are the unique positive solutions. Finally, in the same paper, for $p \in(1,3)$, solutions are found as minimizers on a $L^{2}$-sphere: the value $\omega$ comes out as a Lagrange multiplier and it is not controlled.

Later, the result for $p \in(1,3)$ has been extended by Pomponio and Ruiz [19] by investigating the geometry of the Euler-Lagrange functional. Through a careful analysis for a limit equation, they showed that there exist $0<\omega_{0}<$ $\tilde{\omega}<\bar{\omega}$ such that if $\omega>\bar{\omega}$, the unique solutions to (1.1) are the trivial ones; if $\omega_{0}<\omega<\tilde{\omega}$, there are at least two positive solutions to (1.1); if $0<\omega<\omega_{0}$, there is a positive solution to (1.1) for almost every $\omega$.

Moreover Pomponio and Ruiz also study in [20] the case of a bounded domain for $p \in(1,3)$ and obtain some results on boundary concentration of solutions.

Finally we mention the paper of Wan and Tan [23] where they consider $g(u)=f(u)-\omega u$ with $f$ asymptotically linear and the paper of Huh [11] where infinitely many solutions (possibly sign-changing) have been found for $p>5$.

Remark 1.2. The fact that we obtain nontrivial solutions only for sufficiently small $q$ is not surprising and we can not expect more. Indeed, in [19] the equation

$$
-\Delta u+\omega u+2 u \int_{|x|}^{\infty} \frac{u^{2}(s)}{s} h_{u}(s) d s+u \frac{h_{u}^{2}(|x|)}{|x|^{2}}=|u|^{p-1} u, \quad \text { in } \mathbb{R}^{2}
$$

is considered. Performing the rescaling $u \mapsto u_{\omega}=\omega^{\frac{1}{p-1}} u\left(\omega^{\frac{1}{2}} \cdot\right)$ and defining $q=\omega^{\frac{2(3-p)}{p-1}}$, Eq. (1.4) becomes (1.1), with $g(u)=-u+|u|^{p-1} u$. By [19, Theorem 1.2], for $p \in(1,3)$, there is a solution of (1.1) with $g(u)=-u+|u|^{p-1} u$ only for sufficiently small $q$. Hence, by means of Theorem 1.1, we have now a more precise picture on the existence of solutions of (1.4). There exist, indeed, $\omega_{0}<\tilde{\omega}<\bar{\omega}$ and a decreasing sequence $\left(\omega_{n}\right)_{n \geqslant 1}$ such that the following happens

\begin{tabular}{|l|l|l|l|}
\hline $\begin{array}{l}\text { for all } \\
\omega \in\left(\omega_{n+1}, \omega_{n}\right)\end{array}$ & $\begin{array}{l}\text { for a.e. } \\
\omega \in\left(\omega_{1}, \omega_{0}\right)\end{array}$ & $\begin{array}{l}\text { for all } \\
\omega \in\left(\omega_{0}, \tilde{\omega}\right)\end{array}$ & $\begin{array}{l}\text { for } \\
\omega>\bar{\omega}\end{array}$ \\
\hline $\begin{array}{l}\text { At least } \\
n \text { (possibly sign- } \\
\text { changing) solutions }\end{array}$ & $\begin{array}{l}\text { At least a } \\
\text { positive solution }\end{array}$ & $\begin{array}{l}\text { at least two } \\
\text { positive solutions }\end{array}$ & $\begin{array}{l}\text { no nontrivial } \\
\text { solutions }\end{array}$ \\
\hline
\end{tabular}

The paper is organized as follows. In Sect. 2, for the sake of completeness, we derive the Eq. (1.1). It is obtained by the complete set of the EulerLagrange equations of the Lagrangian density given e.g. in [12, eqn. (8)] and by considering a more general nonlinearity, under a suitable ansatz. Then Sect. 3 is devoted to prove Theorem 1.1. 
Throughout the paper, we denote by $C$ generic positive constants specifying, if necessary, the parameters on which they depend. The constants may also change from line to line.

\section{Deduction of the equation}

Let us consider the three dimensional Lorentz space time $\mathbb{R}^{1,2}$ with metric tensor $\operatorname{diag}(+1,-1,-1)$ and coordinates $x^{\mu}=\left(c t, x^{1}, x^{2}\right)$, where $c$ is the speed of light. As usual, we adopt the Einstein convention on repeated indices, where greek indices always vary in $\{0,1,2\}$ while latin ones run in $\{1,2\}$. In particular we will always distinguish in the following between covariant and contravariant indices, which are obtained ones from the others by using the metric.

The starting point for our equation is the Schrödinger Lagrangian density of the matter field

$$
\mathcal{L}_{\mathrm{S}}(\psi)=i \hbar \bar{\psi} \partial_{t} \psi-\frac{\hbar^{2}}{2 m}|\nabla \psi|^{2}+2 W(\psi)
$$

where $\psi: \mathbb{R}^{1,2} \rightarrow \mathbb{C}, m>0$ is the mass parameter, $\hbar=h / 2 \pi$ is the normalized Planck constant and $W: \mathbb{C} \rightarrow \mathbb{R}$ is a nonlinearity of type $W(\psi)=W(|\psi|)$ which describes the interaction among many particles.

Let us define the electromagnetic tensor as

$$
F_{\mu \nu}=\partial_{\mu} A_{\nu}-\partial_{\nu} A_{\mu}
$$

where $A^{\mu}=\left(A^{0}, \mathbf{A}\right)=\left(A^{0}, A^{1}, A^{2}\right)$ is the gauge potential. We observe explicitly that, in virtue of the choice of the metric,

$$
\left(A^{0}, A^{1}, A^{2}\right)=\left(A_{0},-A_{1},-A_{2}\right)
$$

and indeed we will use, case by case, the notation which is more convenient. In vectorial notation the electromagnetic field is given by

$$
\mathbf{E}=-\nabla A^{0}-\frac{1}{c} \partial_{t} \mathbf{A}, \quad B=\nabla \times \mathbf{A} .
$$

To study the interaction between the matter (expressed by the wave function $\psi$ ) and the electromagnetic field $(\mathbf{E}, B)$ given by (2.3), we consider the gauge (or Weyl) covariant derivatives

$$
D_{t}=\partial_{t}+\frac{i e}{\hbar} A^{0}, \quad \mathbf{D}=\nabla-\frac{i e}{\hbar c} \mathbf{A},
$$

where $e$ is a coupling constant. We point out that these operators are obtained by the so called minimal coupling rule, see e.g. $[8,18]$. Then we substitute in (2.1) the derivatives with the covariant ones, getting

$$
\tilde{\mathcal{L}}_{\mathrm{S}}\left(\psi, A^{0}, \mathbf{A}\right)=i \hbar \bar{\psi} D_{t} \psi-\frac{\hbar^{2}}{2 m}|\mathbf{D} \psi|^{2}+2 W(\psi) .
$$

However to obtain the complete Lagrangian we need to consider also the term involving the gauge potentials, since they are unknown. In $\mathbb{R}^{1,2}$ one can take the more general term 


$$
\mathcal{L}_{\mathrm{MCS}}=\underbrace{-\frac{1}{4} F^{\mu \nu} F_{\mu \nu}}_{\mathcal{L}_{\mathrm{Max}}}+\underbrace{\frac{\kappa}{4} \varepsilon^{\mu \alpha \beta} A_{\mu} F_{\alpha \beta}}_{\mathcal{L}_{\mathrm{CS}}}
$$

which involves not only the usual Maxwell term but also the so-called ChernSimons term. Here $\varepsilon$ is the Levi-Civita tensor and $\kappa \in \mathbb{R}$ is a parameter which controls the Chern-Simons term. Strictly speaking (2.4) is the Lagrangian of the gauge potentials in the vacuum.

Thus the total Lagrangian density is

$$
\begin{aligned}
\mathcal{L}_{\text {tot }}\left(\psi, A^{0}, \mathbf{A}\right) & :=\mathcal{L}_{\mathrm{MCS}}\left(A^{0}, \mathbf{A}\right)+\tilde{\mathcal{L}}_{\mathrm{S}}\left(\psi, A^{0}, \mathbf{A}\right) \\
& =-\frac{1}{4} F^{\mu \nu} F_{\mu \nu}+\frac{\kappa}{4} \varepsilon^{\mu \alpha \beta} A_{\mu} F_{\alpha \beta}+i \hbar \bar{\psi} D_{t} \psi-\frac{\hbar^{2}}{2 m}|\mathbf{D} \psi|^{2}+2 W(\psi) .
\end{aligned}
$$

Due to the presence of the Chern-Simon term, the total Lagrangian is not invariant with respect to the gauge transformations

$$
A^{\mu} \rightarrow A^{\mu}+\partial^{\mu} \chi, \quad \psi \rightarrow \psi e^{-\frac{i e}{\hbar c} \chi}, \quad \chi \in C^{\infty}\left(\mathbb{R}^{1,2}\right),
$$

nevertheless, its Euler-Lagrange equations are gauge invariant.

As discussed in [12], the Chern-Simons electrodynamics is obtained by taking the formal limit $|\kappa| \rightarrow \infty$ in the topologically massive model. Indeed at large distances and low energies the lower derivatives of the Chern-Simons term dominate the higher derivative appearing in the Maxwell term; hence this last term becomes negligible and the above total Lagrangian reduces to

$$
\mathcal{L}\left(\psi, A^{0}, \mathbf{A}\right)=\frac{\kappa}{4} \varepsilon^{\mu \alpha \beta} A_{\mu} F_{\alpha \beta}+i \hbar \bar{\psi} D_{t} \psi-\frac{\hbar^{2}}{2 m}|\mathbf{D} \psi|^{2}+2 W(\psi) .
$$

For this and further discussions see also [7,9,13,21,22]. This is the Lagrangian we are interested in. By taking the variations of the action functional $\mathcal{S}=$ $\iint \mathcal{L} d x d t$ with respect to $\psi, A_{\mu}$, recalling (2.2), we have the following EulerLagrange equations

$$
\begin{aligned}
& i \hbar D_{t} \psi+\frac{\hbar^{2}}{2 m} \mathbf{D}^{2} \psi=-W^{\prime}(\psi) \\
& \kappa\left(\partial_{1} A_{2}-\partial_{2} A_{1}\right)=e|\psi|^{2} \\
& \kappa\left(\partial_{2} A_{0}-\partial_{0} A_{2}\right)=\frac{e}{c} \frac{\hbar}{m} \mathfrak{I m}\left(\bar{\psi} D_{1} \psi\right) \\
& \kappa\left(\partial_{0} A_{1}-\partial_{1} A_{0}\right)=\frac{e}{c} \frac{\hbar}{m} \mathfrak{I m}\left(\bar{\psi} D_{2} \psi\right) .
\end{aligned}
$$

Note that these equations are invariant under the gauge transformations $(2.5)$.

If we define

$$
J^{\mu}=(c \rho, \mathbf{J}):=\left(c \bar{\psi} \psi, \frac{\hbar}{2 m i}(\bar{\psi} \mathbf{D} \psi-\psi \overline{\mathbf{D} \psi})\right)=\left(c|\psi|^{2}, \frac{\hbar}{m} \mathfrak{I m}(\bar{\psi} \mathbf{D} \psi)\right),
$$

the last three equations in (2.6) can be written, since $\mathfrak{I m}\left(\bar{\psi} D^{j} \psi\right)=\mathfrak{I m}\left(\bar{\psi} D_{j} \psi\right)$, as

$$
\frac{\kappa}{2} \varepsilon^{\mu \alpha \beta} F_{\alpha \beta}=\frac{e}{c} J^{\mu}
$$


which are the gauge field equations of the Chern-Simons electrodynamics. Thus, $J^{\mu}$ can be interpreted as a current density. In particular, we have the continuity equation for the currents

$$
\partial_{\mu} J^{\mu}=\partial_{t} \rho+\nabla \cdot \mathbf{J}=0 .
$$

Moreover, in terms of the electromagnetic field (E, B), Eq. (2.7) are

$$
\kappa c B=-e J^{0}, \quad \kappa c E^{1}=e J^{2}, \quad \kappa c E^{2}=-e J^{1} .
$$

The first equation yields the remarkable fact that $B=0$ if and only if $\psi=0$ and that any field configuration with total charge $Q(t)=e \int|\psi(t, x)|^{2} d x$ also carries a magnetic flux $\Phi(t)=\int B(t, x) d x$ given by

$$
\Phi(t)=-\frac{1}{\kappa} Q(t),
$$

and indeed they are conserved quantities (i.e. constant in time) for "well behaved" $\psi$. Moreover the tying in (2.8) provides an explicit realization of anyons, see $[17,24]$. On the other hand, the other two equations give the interesting identities

$$
\nabla \cdot \mathbf{E}=\frac{e}{c \kappa} \nabla \times \mathbf{J} \quad \text { and } \quad \nabla \cdot \mathbf{J}=-\frac{c \kappa}{e} \nabla \times \mathbf{E}
$$

so the matter and the electromagnetic field support each other.

However, it is convenient to write $\psi(t, x)=u(t, x) e^{i S(t, x)}$; hence by taking the variations of the action with respect to $u, S, A_{\mu}$ we get the following set of equations

$$
\begin{aligned}
& -\frac{\hbar^{2}}{2 m} \Delta u+\left(\hbar \partial_{t} S+e A^{0}+\frac{\hbar^{2}}{2 m}|\nabla S|^{2}+\frac{e^{2}}{2 m c^{2}}|\mathbf{A}|^{2}-\frac{\hbar e}{m c} \nabla S \cdot \mathbf{A}\right) u=W^{\prime}(u) \\
& \partial_{t} u^{2}+\frac{\hbar}{m} \nabla \cdot\left[\left(\nabla S-\frac{e}{\hbar c} \mathbf{A}\right) u^{2}\right]=0 \\
& \kappa\left(\partial_{1} A_{2}-\partial_{2} A_{1}\right)=e u^{2} \\
& \kappa\left(\partial_{2} A_{0}-\partial_{0} A_{2}\right)=\frac{e \hbar}{c m}\left(\partial_{1} S+\frac{e}{\hbar c} A_{1}\right) u^{2} \\
& \kappa\left(\partial_{0} A_{1}-\partial_{1} A_{0}\right)=\frac{e \hbar}{c m}\left(\partial_{2} S+\frac{e}{\hbar c} A_{2}\right) u^{2} .
\end{aligned}
$$

The second equation is of course a continuity equation (conservation law of currents), as $\mathcal{L}$ does not depend explicitly on $S$, but just on its derivatives.

In the static case $A^{\mu}=A^{\mu}(x)$, by using the Helmholtz decomposition and taking a suitable gauge, without loss of generality we can assume that $\lim _{|x| \rightarrow+\infty} A^{0}(x)=0$ (if we suppose that such a limit exists) and that $\partial_{j} A^{j}=$ 0 (Coulomb gauge). An interesting case appears when we take the ansatz of standing waves solutions $\psi(t, x)=u(x) e^{i \omega t}$. Then the previous Eq. (2.9) become

$$
\begin{aligned}
& -\frac{\hbar^{2}}{2 m} \Delta u+\left(\hbar \omega+e A^{0}+\frac{e^{2}}{2 m c^{2}}|\mathbf{A}|^{2}\right) u=W^{\prime}(u) \\
& \mathbf{A} \cdot \nabla u^{2}=0 \\
& \kappa\left(\partial_{1} A_{2}-\partial_{2} A_{1}\right)=e u^{2} \\
& \kappa \partial_{2} A_{0}=\frac{e^{2}}{m c^{2}} A_{1} u^{2} \\
& \kappa \partial_{1} A_{0}=-\frac{e^{2}}{m c^{2}} A_{2} u^{2} .
\end{aligned}
$$


Arguing as in [11], the Coulomb gauge and the third equation in (2.10) imply that $A_{1}, A_{2}$ are uniquely determined by $u$, since they satisfy

$$
\Delta A_{1}=-\frac{e}{\kappa} \partial_{2} u^{2}, \quad \Delta A_{2}=\frac{e}{\kappa} \partial_{1} u^{2} .
$$

Hence they are given by

$$
A_{1}=-\frac{e}{\kappa} G_{2} * u^{2}, \quad A_{2}=\frac{e}{\kappa} G_{1} * u^{2}, \quad \text { where } \quad G_{i}(x)=\frac{1}{2 \pi} \frac{x^{i}}{|x|^{2}} .
$$

Coming back to the last two equations in (2.10) we infer that

$$
-\Delta A_{0}=\frac{e^{2}}{\kappa m c^{2}}\left[\partial_{1}\left(A_{2} u^{2}\right)-\partial_{2}\left(A_{1} u^{2}\right)\right]
$$

and hence

$$
A_{0}=\frac{e^{2}}{\kappa m c^{2}}\left[G_{2} *\left(A_{1} u^{2}\right)-G_{1} *\left(A_{2} u^{2}\right)\right] .
$$

Observe also that from the second equation in (2.10) it follows that, up to the "trivial cases", the function $u$ is radial if and only if $\mathbf{A}$ is a tangential vector field, i.e. of type

$$
\mathbf{A}=\frac{e}{\kappa} h_{u}(x) \mathbf{t}, \quad \text { where } \mathbf{t}=\left(x^{2} /|x|^{2},-x^{1} /|x|^{2}\right) .
$$

Moreover, since the problem is invariant by translations, to avoid the related difficulties, we look for radial solutions $u$. Thus, from this choice, arguing as in [11, Lemma 3.3], it follows that $\mathbf{A}$ has to be invariant for the group action

$$
\mathrm{T}_{g} \mathbf{A}(x)=g^{-1} \cdot \mathbf{A}(g(x)), \quad g \in O(2),
$$

and this readily implies that $h_{u}$ has to be a radial function. Summing up, whenever $u$ is radial, the magnetic potential has to be necessarily written as

$$
A^{1}(x)=\frac{e}{\kappa} \frac{x^{2}}{|x|^{2}} h_{u}(|x|), \quad A^{2}(x)=-\frac{e}{\kappa} \frac{x^{1}}{|x|^{2}} h_{u}(|x|) .
$$

Finally, by (2.10) we see that

$$
\nabla A^{0}=-\frac{e^{3}}{m c^{2} \kappa^{2}} u^{2}(|x|) h_{u}(|x|) \mathbf{n}, \quad \text { where } \quad \mathbf{n}=\left(x^{1} /|x|^{2}, x^{2} /|x|^{2}\right)
$$

in other words, the electric potential is radial and so can be written as

$$
A^{0}(x)=A^{0}(|x|) \text {. }
$$

Now we can find the explicit dependence of $A^{0}$ and $h_{u}$ from the function $u$; indeed, by substituting (2.11) and (2.12) into (2.10) one find (assuming $h_{u}(0)=0$, which is necessary to have $\mathbf{A}$ smooth)

$$
h_{u}(|x|)=\int_{0}^{|x|} \tau u^{2}(\tau) d \tau, \quad A^{0}(|x|)=\frac{e^{3}}{m c^{2} \kappa^{2}} \int_{|x|}^{\infty} \frac{u^{2}(\tau)}{\tau} h_{u}(\tau) d \tau .
$$

With these expressions in hands the first equation in (2.10), the equation of the matter field, is

$$
-\frac{\hbar^{2}}{2 m} \Delta u+\hbar \omega u+\frac{e^{4}}{m c^{2} \kappa^{2}} u \int_{|x|}^{\infty} \frac{u^{2}(s)}{s} h_{u}(s) d s+\frac{e^{4}}{2 m c^{2} \kappa^{2}} u \frac{h_{u}^{2}(|x|)}{|x|^{2}}=W^{\prime}(u),
$$


which is nothing but (1.1), "normalizing" the constants $\hbar$ and $2 m$ and taking

$$
q:=\frac{e^{4}}{c^{2} \kappa^{2}}, \quad g(u):=W^{\prime}(u)-\omega u
$$

\section{Proof of Theorem 1.1}

\subsection{Preliminaries}

Following [10], without loss of generality we can replace condition (g1) with

(g1') $\limsup _{\xi \rightarrow \infty} \frac{g(\xi)}{e^{\alpha \xi^{2}}}=0$, for all $\alpha>0$.

Define

$$
m_{0}:=-\frac{1}{2} \limsup _{\xi \rightarrow 0} \frac{g(\xi)}{\xi}>0
$$

and equip $H_{r}^{1}\left(\mathbb{R}^{2}\right)$ with the norm $\|\cdot\|^{2}=\|\nabla \cdot\|_{2}^{2}+m_{0}\|\cdot\|_{2}^{2}$.

Consider $p_{0} \in(1, \infty)$ and set

$$
\begin{gathered}
\lambda(\xi)=\left\{\begin{array}{ll}
\max \left\{g(\xi)+m_{0} \xi, 0\right\}, & \text { for } \xi \geqslant 0, \\
-\lambda(-\xi), & \text { for } \xi<0,
\end{array} \quad \Lambda(\xi)=\int_{0}^{\xi} \lambda(\tau) d \tau,\right. \\
\bar{\lambda}(\xi)=\left\{\begin{array}{ll}
\xi^{p_{0}} \sup _{0<\tau \leqslant \xi} \frac{\lambda(\tau)}{\tau^{p_{0}},} & \text { for } \xi>0, \\
0, & \text { for } \xi=0, \\
-\bar{\lambda}(-\xi), & \text { for } \xi<0,
\end{array} \quad \bar{\Lambda}(\xi)=\int_{0}^{\xi} \bar{\lambda}(\tau) d \tau .\right.
\end{gathered}
$$

The functions $\lambda, \Lambda, \bar{\lambda}$ and $\bar{\Lambda}$ satisfy the following properties (see [10, Lemma 2.1, Corollary 2.2, Lemma 2.3]).

Lemma 3.1. The following holds

(i) $g(\xi)+m_{0} \xi \leqslant \lambda(\xi) \leqslant \bar{\lambda}(\xi)$, for all $\xi \geqslant 0$.

(ii) $\lambda(\xi) \geqslant 0$ and $\bar{\lambda}(\xi) \geqslant 0$, for all $\xi \geqslant 0$.

(iii) There exists $\delta_{0}>0$ such that $\lambda(\xi)=\bar{\lambda}(\xi)=0$ for $\xi \in\left[0, \delta_{0}\right]$.

(iv) There exists $\xi_{0}>0$ such that $0<\lambda\left(\xi_{0}\right) \leqslant \bar{\lambda}\left(\xi_{0}\right)$.

(v) The map $\xi \mapsto \bar{\lambda}(\xi) / \xi^{p_{0}}:(0, \infty) \rightarrow \mathbb{R}$ is non-decreasing.

(vi) $\lambda(\xi), \bar{\lambda}(\xi)$ satisfy (g1').

Corollary 3.2. The following holds

(i) $G(\xi)+m_{0}|\xi|^{2} / 2 \leqslant \Lambda(\xi) \leqslant \bar{\Lambda}(\xi)$, for all $\xi \in \mathbb{R}$.

(ii) $\Lambda(\xi), \bar{\Lambda}(\xi) \geqslant 0$, for all $\xi \in \mathbb{R}$.

(iii) There exists $\delta_{0}>0$ such that $\Lambda(\xi)=\bar{\Lambda}(\xi)=0$ for $\xi \leqslant \delta_{0}$.

(iv) $\bar{\Lambda}\left(\zeta_{0}\right)-m_{0} \zeta_{0}^{2} / 2>0$.

(v) $0 \leqslant\left(p_{0}+1\right) \bar{\Lambda}(\xi) \leqslant \xi \bar{\lambda}(\xi)$, for all $\xi \in \mathbb{R}$.

(vi) $\Lambda(\xi), \bar{\Lambda}(\xi)$ satisfy $\left(g 1^{\prime}\right)$.

Lemma 3.3. Suppose that $u_{j} \rightarrow u_{0}$ in $H_{r}^{1}\left(\mathbb{R}^{2}\right)$, then

(i) $\int_{\mathbb{R}^{2}} \Lambda\left(u_{j}\right) d x \rightarrow \int_{\mathbb{R}^{2}} \Lambda\left(u_{0}\right) d x, \int_{\mathbb{R}^{2}} \bar{\Lambda}\left(u_{j}\right) d x \rightarrow \int_{\mathbb{R}^{2}} \bar{\Lambda}\left(u_{0}\right) d x$; 
(ii) $\lambda\left(u_{j}\right) \rightarrow \lambda\left(u_{0}\right), \bar{\lambda}\left(u_{j}\right) \rightarrow \bar{\lambda}\left(u_{0}\right)$ strongly in $H_{r}^{-1}\left(\mathbb{R}^{2}\right)$.

Now we recall some useful properties of the nonlocal term of the functional $J_{q}$ defined in (1.2). For any $u \in H_{r}^{1}\left(\mathbb{R}^{2}\right)$, let

$$
N(u):=\int_{\mathbb{R}^{2}} \frac{u^{2}(|x|)}{|x|^{2}}\left(\int_{0}^{|x|} s u^{2}(s) d s\right)^{2} d x .
$$

We explicitly remark that

$$
N(u) \leqslant C \int_{\mathbb{R}^{2}} u^{2}(|x|)\left(\int_{B(0,|x|)} u^{4}(y) d y\right) d x \leqslant C\|u\|^{6}
$$

and, for any $v \in H_{r}^{1}\left(\mathbb{R}^{2}\right)$,

$$
\begin{aligned}
N^{\prime}(u)[v]= & 2 \int_{\mathbb{R}^{2}} \frac{u(|x|) v(|x|)}{|x|^{2}}\left(\int_{0}^{|x|} s u^{2}(s) d s\right)^{2} d x \\
& +4 \int_{\mathbb{R}^{2}} \frac{u^{2}(|x|)}{|x|^{2}}\left(\int_{0}^{|x|} s u^{2}(s) d s\right)\left(\int_{0}^{|x|} s u(s) v(s) d s\right) d x .
\end{aligned}
$$

In particular,

$$
N^{\prime}(u)[u]=6 \int_{\mathbb{R}^{2}} \frac{u^{2}(|x|)}{|x|^{2}}\left(\frac{1}{2 \pi} \int_{B(0,|x|)} u^{2}(y) d y\right)^{2} d x=6 N(u) .
$$

Moreover, it is easy to see that the nonlocal term $N(u)$ has the following rescaling properties

$N(u(\tau \cdot))=\tau^{-4} N(u(\cdot))$ and $N^{\prime}(u(\tau \cdot))[v(\tau \cdot)]=\tau^{-4} N^{\prime}(u(\cdot))[v(\cdot)]$ for all $\tau>0$.

We will make use of this property throughout the paper without any other comment.

By using the compact embedding of $H_{r}^{1}\left(\mathbb{R}^{2}\right)$ into $L^{p}\left(\mathbb{R}^{2}\right)$ for $p>2$, we get the following compactness properties of $N(u)$ (see [6, Lemma 3.2]).

Lemma 3.4. If $\left(w_{j}\right)_{j}$ converges weakly to $w$ in $H_{r}^{1}\left(\mathbb{R}^{2}\right)$ as $j \rightarrow \infty$, then, up to a subsequence, the following convergences hold

$$
\begin{aligned}
& N\left(w_{j}\right) \rightarrow N(w), \\
& N^{\prime}\left(w_{j}\right)\left[w_{j}\right] \rightarrow N^{\prime}(w)[w], \\
& N^{\prime}\left(w_{j}\right)[v] \rightarrow N^{\prime}(w)[v], \text { for all } v \in H_{r}^{1}\left(\mathbb{R}^{2}\right) .
\end{aligned}
$$

\subsection{The minimax scheme}

Due to the presence of the nonlocal term and of a nonlinearity satisfying very general assumptions, there are several problems related to the geometry and the compactness of the functional $J_{q}$. So, we are not able to find solutions of 
(1.1) directly and we have to modify the functional by means of a truncation. Therefore, let $\varphi \in C^{\infty}\left(\mathbb{R}_{+},[0,1]\right)$ satisfy

$$
\begin{cases}\varphi(s)=1, & \text { for } s \in[0,1], \\ \varphi(s)=0, & \text { for } s \in[2, \infty), \\ \left\|\varphi^{\prime}\right\|_{\infty} \leqslant 2 & \end{cases}
$$

and consider the perturbed functional $\mathcal{J}_{q}: H_{r}^{1}\left(\mathbb{R}^{2}\right) \rightarrow \mathbb{R}$

$$
\mathcal{J}_{q}(u)=\frac{1}{2}\|\nabla u\|_{2}^{2}+\frac{q}{2} \varphi(q N(u)) N(u)-\int_{\mathbb{R}^{2}} G(u) d x
$$

which is $C^{1}$ and

$$
\begin{aligned}
\mathcal{J}_{q}^{\prime}(u)[v]= & \int_{\mathbb{R}^{2}} \nabla u \nabla v d x+\frac{q^{2}}{2} \varphi^{\prime}(q N(u)) N(u) N^{\prime}(u)[v] \\
& +\frac{q}{2} \varphi(q N(u)) N^{\prime}(u)[v]-\int_{\mathbb{R}^{2}} g(u) v d x
\end{aligned}
$$

We are going to find critical points for $\mathcal{J}_{q}$. Evidently, if we have a bound of type $N(u) \leqslant 1 / q$ on the critical points of $\mathcal{J}_{q}$, then they will be critical points of $J_{q}$ and hence solutions of the Eq. (1.1). Moreover, let us define the following comparison functional

$$
\mathcal{I}(u):=\frac{1}{2}\|\nabla u\|_{2}^{2}-\int_{\mathbb{R}^{2}} \bar{\Lambda}(u) d x
$$

Observe that $\mathcal{J}_{q} \geqslant \mathcal{I}$ [by (i) of Corollary 3.2] and they are both even functionals.

As stated in the next lemma, $\mathcal{J}_{q}$ and $\mathcal{I}$ have the geometry of the Symmetric Mountain Pass Theorem. In what follows, we set $\mathbb{D}_{n}=\left\{\sigma \in \mathbb{R}^{n}:|\sigma| \leqslant 1\right\}$ and $\mathbb{S}^{n-1}=\partial \mathbb{D}_{n}$.

Lemma 3.5. For all $q>0$, the functionals $\mathcal{J}_{q}$ and $\mathcal{I}$ satisfy the following properties.

(i) There exist $r_{0}, \rho_{0}>0$ such that

$$
\begin{gathered}
\mathcal{J}_{q}(u) \geqslant \mathcal{I}(u) \geqslant 0, \text { for }\|u\| \leqslant r_{0}, \\
\mathcal{J}_{q}(u) \geqslant \mathcal{I}(u) \geqslant \rho_{0}, \text { for }\|u\|=r_{0} .
\end{gathered}
$$

(ii) For every $n \in \mathbb{N}$ there exists an odd and continuous map $\gamma_{n}: \mathbb{S}^{n-1} \rightarrow$ $H_{r}^{1}\left(\mathbb{R}^{2}\right)$ such that

$$
\mathcal{I}\left(\gamma_{n}(\sigma)\right) \leqslant \mathcal{J}_{q}\left(\gamma_{n}(\sigma)\right)<0
$$

Proof. The first part follows by [10, Lemma 2.4]. To prove (3.2), we argue as in $\left[4\right.$, Theorem 10]: for any $n \in \mathbb{N}$, an odd and continuous map $\pi_{n}: \mathbb{S}^{n-1} \rightarrow$ $H_{r}^{1}\left(\mathbb{R}^{2}\right)$ is defined such that

$$
0 \notin \pi_{n}\left(\mathbb{S}^{n-1}\right) \text { and } \int_{\mathbb{R}^{2}} G\left(\pi_{n}(\sigma)\right) d x \geqslant 1, \quad \text { for all } \sigma \in \mathbb{S}^{n-1} \text {. }
$$


Then for $\theta$ sufficiently large, setting $\gamma_{n}(\sigma):=\pi_{n}(\sigma)(\cdot / \theta)$, we have

$$
\begin{aligned}
\mathcal{J}_{q}\left(\gamma_{n}(\sigma)\right)= & \frac{1}{2} \int_{\mathbb{R}^{2}}\left|\nabla \pi_{n}(\sigma)\right|^{2} d x+\frac{q}{2} \theta^{4} \varphi\left(q \theta^{4} N\left(\pi_{n}(\sigma)\right)\right) N\left(\pi_{n}(\sigma)\right) \\
& -\theta^{2} \int_{\mathbb{R}^{2}} G\left(\pi_{n}(\sigma)\right) d x \\
\leqslant & \frac{1}{2} \int_{\mathbb{R}^{2}}\left|\nabla \pi_{n}(\sigma)\right|^{2} d x-\theta^{2}<0
\end{aligned}
$$

Due to Lemma 3.5 , for every $n \in \mathbb{N}$, we can define a family of mappings $\Gamma_{n}$ by

$$
\Gamma_{n}:=\left\{\gamma \in C\left(\mathbb{D}_{n}, H_{r}^{1}\left(\mathbb{R}^{2}\right)\right): \gamma(-\sigma)=-\gamma(\sigma) \text { and } \gamma_{\mid \mathbb{S}^{n-1}}=\gamma_{n}\right\},
$$

which is nonempty since

$$
\alpha_{n}(\sigma)= \begin{cases}0, & \text { for } \sigma=0, \\ |\sigma| \gamma_{n}(\sigma /|\sigma|), & \text { for } \sigma \in \mathbb{D}_{n} \backslash\{0\},\end{cases}
$$

belongs to $\Gamma_{n}$.

Now define the values

$$
b_{n}(q):=\inf _{\gamma \in \Gamma_{n}} \max _{\sigma \in \mathbb{D}_{n}} \mathcal{J}_{q}(\gamma(\sigma)) .
$$

A first property of these levels is the following estimate.

Lemma 3.6. For all $q>0$ and $n \in \mathbb{N}$, there exists a constant $C(n)$, such that $b_{n}(q) \leqslant C(n)$.

Proof. For a fixed $\gamma \in \Gamma_{n}$, we have

$$
\begin{aligned}
b_{n}(q) & \leqslant \max _{\sigma \in \mathbb{D}_{n}} \mathcal{J}_{q}(\gamma(\sigma)) \\
& \leqslant \max _{\sigma \in \mathbb{D}_{n}}\left\{\frac{1}{2}\|\nabla \gamma(\sigma)\|_{2}^{2}-\int G(\gamma(\sigma))\right\}+\max _{\sigma \in \mathbb{D}_{n}}\left\{\frac{q}{2} \varphi(q N(\gamma(\sigma))) N(\gamma(\sigma))\right\} \\
& \leqslant \begin{cases}C(n), & \text { if } N(\gamma(\sigma)) \geqslant 2 / q \\
C(n)+1, & \text { if } N(\gamma(\sigma))<2 / q .\end{cases}
\end{aligned}
$$

Lemma 3.7. For all $q>0$, the values $b_{n}(q)$ are divergent and so we can assume that they are strictly monotone.

Proof. In [10, Lemma 3.2] it has been proved that the values

$$
c_{n}:=\inf _{\gamma \in \Gamma_{n}} \max _{\sigma \in \mathbb{D}_{n}} \mathcal{I}(\gamma(\sigma)) \rightarrow+\infty \quad \text { as } n \rightarrow+\infty .
$$

Moreover it is easy to see that for all $\gamma \in \Gamma_{n}, \gamma\left(\mathbb{D}_{n}\right) \cap\left\{u \in H_{r}^{1}\left(\mathbb{R}^{2}\right):\|u\|=r_{0}\right\}$ $\neq \emptyset$. The conclusion then follows since, by (i) Lemma 3.5,

$$
0<\rho_{0} \leqslant c_{n} \leqslant b_{n}(q) \text {. }
$$


To deal with the lack of compactness, it is convenient to work in the augmented space $\mathbb{R} \times H_{r}^{1}\left(\mathbb{R}^{2}\right)$. For this, we define the extended functional

$$
\begin{gathered}
\tilde{\mathcal{J}}_{q}(\theta, u):=\mathcal{J}_{q}\left(u\left(e^{-\theta} \cdot\right)\right)=\frac{1}{2}\|\nabla u\|_{2}^{2}+\frac{q}{2} e^{4 \theta} \varphi\left(q e^{4 \theta} N(u)\right) N(u) \\
-e^{2 \theta} \int_{\mathbb{R}^{2}} G(u) d x
\end{gathered}
$$

and its derivative will be denoted by $\tilde{\mathcal{J}}_{q}^{\prime}=\left(\partial_{\theta} \tilde{\mathcal{J}}_{q}, \partial_{u} \tilde{\mathcal{J}}_{q}\right)$ with

$$
\begin{aligned}
\partial_{\theta} \tilde{\mathcal{J}}_{q}(\theta, u)= & 2 q e^{4 \theta} \varphi\left(q e^{4 \theta} N(u)\right) N(u)+2 q^{2} e^{8 \theta} \varphi^{\prime}\left(q e^{4 \theta} N(u)\right) N^{2}(u) \\
& -2 e^{2 \theta} \int_{\mathbb{R}^{2}} G(u) d x,
\end{aligned}
$$

and

$$
\begin{aligned}
\partial_{u} \tilde{\mathcal{J}}_{q}(\theta, u)[v]= & \int_{\mathbb{R}^{2}} \nabla u \nabla v d x+\frac{q^{2}}{2} e^{8 \theta} \varphi^{\prime}\left(q e^{4 \theta} N(u)\right) N(u) N^{\prime}(u)[v] \\
& +\frac{q}{2} e^{4 \theta} \varphi\left(q e^{4 \theta} N(u)\right) N^{\prime}(u)[v]-e^{2 \theta} \int_{\mathbb{R}^{2}} g(u) v d x .
\end{aligned}
$$

for all $v \in H_{r}^{1}\left(\mathbb{R}^{2}\right)$.

Let us define the classes

$$
\begin{aligned}
\tilde{\Gamma}_{n}= & \left\{\tilde{\gamma}=(\theta, \eta) \in C\left(\mathbb{D}_{n}, \mathbb{R} \times H_{r}^{1}\left(\mathbb{R}^{2}\right)\right): \theta \text { is even, } \eta\right. \text { is odd, and } \\
& \left.\tilde{\gamma}_{\mid \mathbb{S}^{n-1}}=\left(0, \gamma_{n}\right)\right\}
\end{aligned}
$$

where $\gamma_{n}$ is given in Lemma 3.5, and the levels

$$
\tilde{b}_{n}(q):=\inf _{\gamma \in \tilde{\Gamma}_{n}} \max _{\sigma \in \mathbb{D}_{n}} \tilde{\mathcal{J}}_{q}(\gamma(\sigma)) \text {. }
$$

Arguing as in [10, Lemma 4.1], we have

Lemma 3.8. For all $q>0$, we have $\tilde{b}_{n}(q)=b_{n}(q)$; hence $\tilde{b}_{n}(q) \rightarrow+\infty$.

To show that $b_{n}(q)$ are critical values for $\mathcal{J}_{q}$, we begin by showing that $\tilde{b}_{n}(q)$, i.e. $b_{n}(q)$ by Lemma 3.8 , are "almost critical values" for $\tilde{\mathcal{J}}_{q}$ with a further important property. The proof is similar to [10, Proposition 4.2].

Proposition 3.9. For all $q>0$ and $n \in \mathbb{N}$, there exists a (PS) sequence $\left(\theta_{j}^{(n, q)}, u_{j}^{(n, q)}\right)_{j}$ for $\tilde{\mathcal{J}}_{q}$ at level $b_{n}(q)$ such that $\theta_{j}^{(n, q)} \rightarrow 0$ as $j \rightarrow \infty$.

Thus we are ready to prove the following fundamental result.

Proposition 3.10. Let us fix $n \in \mathbb{N}$. There exists $\bar{q}(n)>0$ such that for every $q \in(0, \bar{q}(n))$, if $\left(\theta_{j}^{(n, q)}, u_{j}^{(n, q)}\right)_{j}$ is a (PS) sequence as in Proposition 3.9 , then $\left(u_{j}^{(n, q)}\right)_{j}$ is bounded in $H_{r}^{1}\left(\mathbb{R}^{2}\right)$ uniformly with respect to q. Furthermore, possibly passing to a subsequence, it converges to a critical point $u^{(n, q)}$ of $\mathcal{J}_{q}$. In particular $b_{n}(q)$ is a critical value for $\mathcal{J}_{q}$. 
Proof. Since $\tilde{\mathcal{J}}_{q}\left(\theta_{j}^{(n, q)}, u_{j}^{(n, q)}\right)=b_{n}(q)+o_{j}(1)$ and $\partial_{\theta} \tilde{\mathcal{J}}_{q}\left(\theta_{j}^{(n, q)}, u_{j}^{(n, q)}\right)=o_{j}(1)$, then

$$
2 \tilde{\mathcal{J}}_{q}\left(\theta_{j}^{(n, q)}, u_{j}^{(n, q)}\right)-\partial_{\theta} \tilde{\mathcal{J}}_{q}\left(\theta_{j}^{(n, q)}, u_{j}^{(n, q)}\right)=2 b_{n}(q)+o_{j}(1),
$$

which is equivalent, by using (3.3) and (3.4), to

$$
\left\|\nabla u_{j}^{(n, q)}\right\|_{2}^{2}=2 b_{n}(q)+C_{j}^{(n, q)}+D_{j}^{(n, q)}+o_{j}(1)
$$

where

$$
\begin{aligned}
& C_{j}^{(n, q)}:=q e^{4 \theta_{j}^{(n, q)}} \varphi\left(q e^{4 \theta_{j}^{(n, q)}} N\left(u_{j}^{(n, q)}\right)\right) N\left(u_{j}^{(n, q)}\right) \\
& D_{j}^{(n, q)}:=2 q^{2} e^{8 \theta_{j}^{(n, q)}} \varphi^{\prime}\left(q e^{4 \theta_{j}^{(n, q)}} N\left(u_{j}^{(n, q)}\right)\right) N^{2}\left(u_{j}^{(n, q)}\right) .
\end{aligned}
$$

We easily see that

$$
\begin{aligned}
& \text { if } q e^{4 \theta_{j}^{(n, q)}} N\left(u_{j}^{(n, q)}\right) \geqslant 2 \text {, then } C_{j}^{(n, q)}=D_{j}^{(n, q)}=0, \\
& \text { if } q e^{4 \theta_{j}^{(n, q)}} N\left(u_{j}^{(n, q)}\right)<2 \text {, then } C_{j}^{(n, q)}<2, D_{j}^{(n, q)}<16,
\end{aligned}
$$

so, in any case, by Lemma 3.6,

$$
\left\|\nabla u_{j}^{(n, q)}\right\|_{2}^{2} \leqslant C(n)
$$

We show now that there exist $\bar{q}(n)>0$ and $C(n)>0$ such that for all $q \in$ $(0, \bar{q}(n))$, there exists $j_{0} \in \mathbb{N}$ such that for all $j \geqslant j_{0},\left\|u_{j}^{(n, q)}\right\|_{2}^{2} \leqslant C(n)$ : this will prove the uniform boundedness of $\left(u_{j}^{(n, q)}\right)_{j}$ in $H_{r}^{1}\left(\mathbb{R}^{2}\right)$.

Arguing by contradiction, let us assume that

$$
\forall k \in \mathbb{N}^{*} \exists q_{k} \in(0,1 / k) \text { s.t. } \forall h \in \mathbb{N} \exists j_{k, h} \geqslant h:\left\|u_{j_{k, h}}^{\left(n, q_{k}\right)}\right\|_{2}^{2}>k .
$$

Let $k=1$ and consider the associated $q_{1}$. By Proposition 3.9, there exists $j^{1} \in \mathbb{N}$ such that $\left|\theta_{j}^{\left(n, q_{1}\right)}\right|<1$ and $\left\|\partial_{u} \tilde{\mathcal{J}}_{q_{1}}\left(\theta_{j}^{\left(n, q_{1}\right)}, u_{j}^{\left(n, q_{1}\right)}\right)\right\|<1$, for all $j \geqslant$ $j^{1}$. Hence, by (3.6), taking $h_{1}=\max \left\{j^{1}, 1\right\}$, there exists $j_{1} \geqslant h_{1}$ such that $\left\|u_{j_{1}}^{\left(n, q_{1}\right)}\right\|_{2}^{2}>1$.

Now let $k=2$ and consider the associated $q_{2}$. By Proposition 3.9, there exists $j^{2}>j_{1}$ such that $\left|\theta_{j}^{\left(n, q_{2}\right)}\right|<1 / 2$ and $\left\|\partial_{u} \tilde{\mathcal{J}}_{q_{2}}\left(\theta_{j}^{\left(n, q_{2}\right)}, u_{j}^{\left(n, q_{2}\right)}\right)\right\|<1 / 2$, for all $j \geqslant j^{2}$. Again, by (3.6), taking $h_{2}=\max \left\{j^{2}, 2\right\}$, there exists $j_{2} \geqslant h_{2}$ such that $\left\|u_{j_{2}}^{\left(n, q_{2}\right)}\right\|_{2}^{2}>2$.

By an iterative procedure, for all $k \in \mathbb{N}$, there exists $j_{k} \geqslant k$ such that

$$
\left|\theta_{j_{k}}^{\left(n, q_{k}\right)}\right|<\frac{1}{k}, \quad\left\|\partial_{u} \tilde{\mathcal{J}}_{q_{k}}\left(\theta_{j_{k}}^{\left(n, q_{k}\right)}, u_{j_{k}}^{\left(n, q_{k}\right)}\right)\right\|<\frac{1}{k}, \quad\left\|u_{j_{k}}^{\left(n, q_{k}\right)}\right\|_{2}^{2}>k .
$$

For the sake of brevity, we rename the sequence that satisfies $(3.7)$ by $\left(\tilde{\theta}_{j}, \tilde{u}_{j}\right)_{j}$. Since $\partial_{u} \tilde{\mathcal{J}}_{q_{j}}\left(\tilde{\theta}_{j}, \tilde{u}_{j}\right) \rightarrow 0$, then for all $v \in H_{r}^{1}\left(\mathbb{R}^{2}\right)$,

$$
\left|\partial_{u} \tilde{\mathcal{J}}_{q_{j}}\left(\tilde{\theta}_{j}, \tilde{u}_{j}\right)[v]\right| \leqslant \varepsilon_{j}\|v\|,
$$

where

$$
\varepsilon_{j}:=\left\|\partial_{u} \tilde{\mathcal{J}}_{q_{j}}\left(\tilde{\theta}_{j}, \tilde{u}_{j}\right)\right\| \rightarrow 0
$$


More precisely, using (3.5),

$$
\begin{aligned}
& \mid \int_{\mathbb{R}^{2}} \nabla \tilde{u}_{j} \nabla v d x+\frac{q_{j}^{2}}{2} e^{8 \tilde{\theta}_{j}} \varphi^{\prime}\left(q_{j} e^{4 \tilde{\theta}_{j}} N\left(\tilde{u}_{j}\right)\right) N\left(\tilde{u}_{j}\right) N^{\prime}\left(\tilde{u}_{j}\right)[v] \\
& \quad+\frac{q_{j}}{2} e^{4 \tilde{\theta}_{j}} \varphi\left(q_{j} e^{4 \tilde{\theta}_{j}} N\left(\tilde{u}_{j}\right)\right) N^{\prime}\left(\tilde{u}_{j}\right)[v]-e^{2 \tilde{\theta}_{j}} \int_{\mathbb{R}^{2}} g\left(\tilde{u}_{j}\right) v d x \mid \\
& \leqslant \varepsilon_{j} \sqrt{\|\nabla v\|_{2}^{2}+m_{0}\|v\|_{2}^{2}} .
\end{aligned}
$$

Consider $t_{j}=1 /\left\|\tilde{u}_{j}\right\|_{2} \rightarrow 0$ as $j \rightarrow \infty$ and $\widehat{u}_{j}(\cdot)=\tilde{u}_{j}\left(\cdot / t_{j}\right)$. Thus we have

$$
\begin{aligned}
& \left\|\widehat{u}_{j}\right\|_{2}^{2}=1, \\
& \left\|\nabla \widehat{u}_{j}\right\|_{2}^{2}=\left\|\nabla \tilde{u}_{j}\right\|_{2}^{2} \leqslant C(n) .
\end{aligned}
$$

Then $\left(\widehat{u}_{j}\right)_{j}$ is bounded in $H_{r}^{1}\left(\mathbb{R}^{2}\right)$ and so, along a subsequence, $\widehat{u}_{j} \rightarrow \widehat{u}_{0}$ weakly in $H_{r}^{1}\left(\mathbb{R}^{2}\right)$.

Claim. $\widehat{u}_{0}=0$.

Let $v \in C_{0}^{\infty}\left(\mathbb{R}^{2}\right)$ and $\widehat{v}(\cdot):=v\left(t_{j} \cdot\right)$. Evaluating (3.8) with $\widehat{v}$ and multiplying by $t_{j}^{2}$, we obtain

$$
\begin{aligned}
& \mid t_{j}^{2} \int_{\mathbb{R}^{2}} \nabla \widehat{u}_{j} \nabla v d x+\frac{q_{j}^{2}}{2} \frac{e^{8 \tilde{\theta}_{j}}}{t_{j}^{6}} \varphi^{\prime}\left(\frac{q_{j} e^{4 \tilde{\theta}_{j}} N\left(\widehat{u}_{j}\right)}{t_{j}^{4}}\right) N\left(\widehat{u}_{j}\right) N^{\prime}\left(\widehat{u}_{j}\right)[v] \\
& \quad+\frac{q_{j}}{2} \frac{e^{4 \tilde{\theta}_{j}}}{t_{j}^{2}} \varphi\left(\frac{q_{j} e^{4 \tilde{\theta}_{j}} N\left(\widehat{u}_{j}\right)}{t_{j}^{4}}\right) N^{\prime}\left(\widehat{u}_{j}\right)[v]-e^{2 \tilde{\theta}_{j}} \int_{\mathbb{R}^{2}} g\left(\widehat{u}_{j}\right) v d x \mid \\
& \leqslant \varepsilon_{j} t_{j} \sqrt{t_{j}^{2}\|\nabla v\|_{2}^{2}+m_{0}\|v\|_{2}^{2}} .
\end{aligned}
$$

Now we distinguish some cases as follows.

Case 1. $q_{j} e^{4 \tilde{\theta}_{j}} N\left(\widehat{u}_{j}\right) \leqslant 2 t_{j}^{4}$.

Case 1.1. If $t_{j}^{4} / q_{j} \rightarrow 0$ then $N\left(\widehat{u}_{j}\right) \rightarrow 0$ as $j \rightarrow \infty$. From [6, Proposition 2.4] and (3.10), we have

$$
\left\|\widehat{u}_{j}\right\|_{4}^{4} \leqslant 2\left\|\nabla \widehat{u}_{j}\right\|_{2} N^{1 / 2}\left(\widehat{u}_{j}\right) \rightarrow 0
$$

and hence we deduce $\widehat{u}_{0} \equiv 0$.

Case 1.2. If $t_{j}^{4} / q_{j} \geqslant C$, by the compactness of $N$ and $N^{\prime}$ (see Lemma 3.4) and the definition of $\varphi$, we infer that

$$
\begin{aligned}
& \left|\frac{q_{j}^{2}}{2} \frac{e^{8 \tilde{\theta}_{j}}}{t_{j}^{6}} \varphi^{\prime}\left(\frac{q_{j} e^{4 \tilde{\theta}_{j}} N\left(\widehat{u}_{j}\right)}{t_{j}^{4}}\right) N\left(\widehat{u}_{j}\right) N^{\prime}\left(\widehat{u}_{j}\right)[v]\right| \leqslant C t_{j}^{2}, \\
& \left|\frac{q_{j}}{2} \frac{e^{4 \tilde{\theta}_{j}}}{t_{j}^{2}} \varphi\left(\frac{q_{j} e^{4 \tilde{\theta}_{j}} N\left(\widehat{u}_{j}\right)}{t_{j}^{4}}\right) N^{\prime}\left(\widehat{u}_{j}\right)[v]\right| \leqslant C t_{j}^{2} .
\end{aligned}
$$

Hence, since $t_{j} \rightarrow 0$ as $j \rightarrow \infty$, by (3.11), we have $\int_{\mathbb{R}^{2}} g\left(\widehat{u}_{0}\right) v d x=0$ for all $v \in C_{0}^{\infty}\left(\mathbb{R}^{2}\right)$. From this and by condition $\left(\mathrm{g} 2{ }^{\prime}\right), \widehat{u}_{0} \equiv 0$.

Case 2. $\frac{q_{j} e^{4 \tilde{\theta}_{j}} N\left(\widehat{u}_{j}\right)}{t_{j}^{4}}>2$. 
Equation (3.11), in this case, becomes

$$
\left|t_{j}^{2} \int_{\mathbb{R}^{2}} \nabla \widehat{u}_{j} \nabla v d x-e^{2 \tilde{\theta}_{j}} \int_{\mathbb{R}^{2}} g\left(\widehat{u}_{j}\right) v d x\right| \leqslant \varepsilon_{j} t_{j} \sqrt{t_{j}^{2}\|\nabla v\|_{2}^{2}+m_{0}\|v\|_{2}^{2}}
$$

and we conclude simply repeating the arguments in Case 1.2 completing so the proof of the claim.

Now evaluating (3.8) in $\tilde{u}_{j}$ and multiplying by $t_{j}^{2}$ we obtain

$$
\begin{aligned}
& t_{j}^{2}\left\|\nabla \widehat{u}_{j}\right\|_{2}^{2}+\frac{q_{j}^{2}}{2} \frac{e^{8 \tilde{\theta}_{j}}}{t_{j}^{6}} \varphi^{\prime}\left(\frac{q_{j} e^{4 \tilde{\theta}_{j}} N\left(\widehat{u}_{j}\right)}{t_{j}^{4}}\right) N\left(\widehat{u}_{j}\right) N^{\prime}\left(\widehat{u}_{j}\right)\left[\widehat{u}_{j}\right] \\
& \quad+\frac{q_{j}}{2} \frac{e^{4 \tilde{\theta}_{j}}}{t_{j}^{2}} \varphi\left(\frac{q_{j} e^{4 \tilde{\theta}_{j}} N\left(\widehat{u}_{j}\right)}{t_{j}^{4}}\right) N^{\prime}\left(\widehat{u}_{j}\right)\left[\widehat{u}_{j}\right]-e^{2 \tilde{\theta}_{j}} \int_{\mathbb{R}^{2}} g\left(\widehat{u}_{j}\right) \widehat{u}_{j} d x=o_{j}(1) .
\end{aligned}
$$

Thus, by Lemmas 3.1 and 3.3 and (3.1)

$$
\begin{aligned}
& t_{j}^{2}\left\|\nabla \widehat{u}_{j}\right\|_{2}^{2}+m_{0} e^{2 \tilde{\theta}_{j}}\left\|\widehat{u}_{j}\right\|_{2}^{2}+3 q_{j}^{2} \frac{e^{8 \tilde{\theta}_{j}}}{t_{j}^{6}} \varphi^{\prime}\left(\frac{q_{j} e^{4 \tilde{\theta}_{j}} N\left(\widehat{u}_{j}\right)}{t_{j}^{4}}\right) N^{2}\left(\widehat{u}_{j}\right) \\
& \quad+3 q_{j} \frac{e^{4 \tilde{\theta}_{j}}}{t_{j}^{2}} \varphi\left(\frac{q_{j} e^{4 \tilde{\theta}_{j}} N\left(\widehat{u}_{j}\right)}{t_{j}^{4}}\right) N\left(\widehat{u}_{j}\right) \\
& =e^{2 \tilde{\theta}_{j}} \int_{\mathbb{R}^{2}}\left(g\left(\widehat{u}_{j}\right) \widehat{u}_{j}+m_{0}\left(\widehat{u}_{j}\right)^{2}\right) d x+o_{j}(1) \leqslant e^{2 \tilde{\theta}_{j}} \int_{\mathbb{R}^{2}} \lambda\left(\widehat{u}_{j}\right) \widehat{u}_{j} d x+o_{j}(1) \rightarrow 0 .
\end{aligned}
$$

Now we show that

$$
3 q_{j}^{2} \frac{e^{8 \tilde{\theta}_{j}}}{t_{j}^{6}} \varphi^{\prime}\left(\frac{q_{j} e^{4 \tilde{\theta}_{j}} N\left(\widehat{u}_{j}\right)}{t_{j}^{4}}\right) N^{2}\left(\widehat{u}_{j}\right) \rightarrow 0 .
$$

If $q_{j} e^{4 \tilde{\theta}_{j}} N\left(\widehat{u}_{j}\right) \geqslant 2 t_{j}^{4}$, then the desired convergence follows easily. On the other hand, if we have $q_{j} e^{4 \tilde{\theta}_{j}} N\left(\widehat{u}_{j}\right)<2 t_{j}^{4}$, then

$$
\left|3 q_{j}^{2} \frac{e^{8 \tilde{\theta}_{j}}}{t_{j}^{6}} \varphi^{\prime}\left(\frac{q_{j} e^{4 \tilde{\theta}_{j}} N\left(\widehat{u}_{j}\right)}{t_{j}^{4}}\right) N^{2}\left(\widehat{u}_{j}\right)\right| \leqslant C t_{j}^{2} \rightarrow 0
$$

Therefore, from (3.12), since

$$
3 q_{j} \frac{e^{4 \tilde{\theta}_{j}}}{t_{j}^{2}} \varphi\left(\frac{q_{j} e^{4 \tilde{\theta}_{j}} N\left(\widehat{u}_{j}\right)}{t_{j}^{4}}\right) N\left(\widehat{u}_{j}\right) \geqslant 0,
$$

we get $\left\|\widehat{u}_{j}\right\|_{2} \rightarrow 0$, which is in contradiction with $(3.9)$. Hence $\left(u_{j}^{(n, q)}\right)_{j}$ is bounded in $H_{r}^{1}\left(\mathbb{R}^{2}\right)$ uniformly with respect to $q$. So, we can assume that there exists $u^{(n, q)} \in H_{r}^{1}\left(\mathbb{R}^{2}\right)$ such that, for $j \rightarrow \infty$,

$$
\begin{aligned}
& u_{j}^{(n, q)} \rightarrow u^{(n, q)} \text { in } H_{r}^{1}\left(\mathbb{R}^{2}\right), \quad u_{j}^{(n, q)} \rightarrow u^{(n, q)} \text { in } L^{p}\left(\mathbb{R}^{2}\right), p>2, \\
& u_{j}^{(n, q)} \rightarrow u^{(n, q)} \text { a.e. in } \mathbb{R}^{2} .
\end{aligned}
$$


Vol. 22 (2015) A multiplicity result for Chern-Simons-Schrödinger...

By hypotheses we know that for any $v \in H_{r}^{1}\left(\mathbb{R}^{2}\right)$,

$\partial_{u} \tilde{\mathcal{J}}_{q}\left(\theta_{j}^{(n, q)}, u_{j}^{(n, q)}\right)[v]=\int_{\mathbb{R}^{2}} \nabla u_{j}^{(n, q)} \nabla v d x$

$$
\begin{aligned}
& +\frac{q^{2}}{2} e^{8 \theta_{j}^{(n, q)}} \varphi^{\prime}\left(q e^{4 \theta_{j}^{(n, q)}} N\left(u_{j}^{(n, q)}\right)\right) N\left(u_{j}^{(n, q)}\right) N^{\prime}\left(u_{j}^{(n, q)}\right)[v] \\
& +\frac{q}{2} e^{4 \theta_{j}^{(n, q)}} \varphi\left(q e^{4 \theta_{j}^{(n, q)}} N\left(u_{j}^{(n, q)}\right)\right) N^{\prime}\left(u_{j}^{(n, q)}\right)[\phi] \\
& -e^{2 \theta_{j}^{(n, q)}} \int_{\mathbb{R}^{2}} g\left(u_{j}^{(n, q)}\right) \phi d x \rightarrow 0
\end{aligned}
$$

as $j \rightarrow \infty$. Then by (3.13) and Lemma 3.4, $u^{(n, q)}$ satisfies

$$
\begin{gathered}
\int_{\mathbb{R}^{2}} \nabla u^{(n, q)} \nabla v d x+\frac{q^{2}}{2} \varphi^{\prime}\left(q N\left(u^{(n, q)}\right)\right) N\left(u^{(n, q)}\right) N^{\prime}\left(u^{(n, q)}\right)[v] \\
+\frac{q}{2} \varphi\left(q N\left(u^{(n, q)}\right)\right) N^{\prime}\left(u^{(n, q)}\right)[v]-\int_{\mathbb{R}^{2}} g\left(u^{(n, q)}\right) v d x=0
\end{gathered}
$$

for every $v \in H_{r}^{1}\left(\mathbb{R}^{2}\right)$. In particular

$$
\begin{aligned}
\left\|u^{(n, q)}\right\|^{2}+\frac{q^{2}}{2} \varphi^{\prime}\left(q N\left(u^{(n, q)}\right)\right) N\left(u^{(n, q)}\right) N^{\prime}\left(u^{(n, q)}\right)\left[u^{(n, q)}\right] \\
+\frac{q}{2} \varphi\left(q N\left(u^{(n, q)}\right)\right) N^{\prime}\left(u^{(n, q)}\right)\left[u^{(n, q)}\right] \\
-\int_{\mathbb{R}^{2}}\left(g\left(u^{(n, q)}\right) u^{(n, q)}+m_{0}\left(u^{(n, q)}\right)^{2}\right) d x=0 .
\end{aligned}
$$

Now, by considering $v=u_{j}^{(n, q)}$ in (3.14), we have

$$
\begin{aligned}
& \left\|\nabla u_{j}^{(n, q)}\right\|^{2}+m_{0} e^{2 \theta_{j}^{(n, q)}}\left\|u_{j}^{(n, q)}\right\|_{2}^{2} \\
& \quad+\frac{q^{2}}{2} e^{8 \theta_{j}^{(n, q)}} \varphi^{\prime}\left(q e^{4 \theta_{j}^{(n, q)}} N\left(u_{j}^{(n, q)}\right)\right) N\left(u_{j}^{(n, q)}\right) N^{\prime}\left(u_{j}^{(n, q)}\right)\left[u_{j}^{(n, q)}\right] \\
& \quad+\frac{q}{2} e^{4 \theta_{j}^{(n, q)}} \varphi\left(q e^{4 \theta_{j}^{(n, q)}} N\left(u_{j}^{(n, q)}\right)\right) N^{\prime}\left(u_{j}^{(n, q)}\right)\left[u_{j}^{(n, q)}\right] \\
& =e^{2 \theta_{j}^{(n, q)}} \int_{\mathbb{R}^{2}} \lambda\left(u_{j}^{(n, q)}\right) u_{j}^{(n, q)} d x \\
& \quad-e^{2 \theta_{j}^{(n, q)}} \int_{\mathbb{R}^{2}}\left(\lambda\left(u_{j}^{(n, q)}\right) u_{j}^{(n, q)}-g\left(u_{j}^{(n, q)}\right) u_{j}^{(n, q)}-m_{0}\left(u_{j}^{(n, q)}\right)^{2}\right) d x+o_{j}(1) .
\end{aligned}
$$

From Lemma 3.1, for every $j \in \mathbb{N}$ and $x \in \mathbb{R}^{2}$,

$$
\lambda\left(u_{j}^{(n, q)}(x)\right) u_{j}^{(n, q)}(x)-g\left(u_{j}^{(n, q)}(x)\right) u_{j}^{(n, q)}(x)-m_{0}\left(u_{j}^{(n, q)}(x)\right)^{2} \geqslant 0 .
$$

Thus, by Fatou's Lemma,

$$
\begin{gathered}
\liminf _{j \rightarrow \infty} \int_{\mathbb{R}^{2}}\left(\lambda\left(u_{j}^{(n, q)}\right) u_{j}^{(n, q)}-m_{0}\left(u_{j}^{(n, q)}\right)^{2}-g\left(u_{j}^{(n, q)}\right) u_{j}^{(n, q)}\right) d x \\
\geqslant \int_{\mathbb{R}^{2}}\left(\lambda\left(u^{(n, q)}\right) u^{(n, q)}-m_{0}\left(u^{(n, q)}\right)^{2}-g\left(u^{(n, q)}\right) u^{(n, q)}\right) d x
\end{gathered}
$$

Due to Lemma 3.3, we have

$$
\int_{\mathbb{R}^{2}} \lambda\left(u_{j}^{(n, q)}\right) u_{j}^{(n, q)} d x \rightarrow \int_{\mathbb{R}^{2}} \lambda\left(u^{(n, q)}\right) u^{(n, q)} d x .
$$


Finally, using (3.15), (3.16), (3.17), (3.18) and Lemma 3.4, we deduce that

$$
\begin{aligned}
\limsup _{j \rightarrow \infty}\left\|u_{j}^{(n, q)}\right\|^{2}= & \limsup _{j \rightarrow \infty}\left[-\frac{q^{2}}{2} e^{8 \theta_{j}^{(n, q)}} \varphi^{\prime}\left(q e^{4 \theta_{j}^{(n, q)}} N\left(u_{j}^{(n, q)}\right)\right) N\left(u_{j}^{(n, q)}\right) N^{\prime}\left(u_{j}^{(n, q)}\right)\left[u_{j}^{(n, q)}\right]\right. \\
& -\frac{q}{2} e^{4 \theta_{j}^{(n, q)}} \varphi\left(e^{4 \theta_{j}^{(n, q)}} N\left(u_{j}^{(n, q)}\right)\right) N^{\prime}\left(u_{j}^{(n, q)}\right)\left[u_{j}^{(n, q)}\right] \\
+ & e^{2 \theta_{j}^{(n, q)}} \int_{\mathbb{R}^{2}} \lambda\left(u_{j}^{(n, q)}\right) u_{j}^{(n, q)} d x \\
& \left.-e^{2 \theta_{j}^{(n, q)}} \int_{\mathbb{R}^{2}}\left(\lambda\left(u_{j}^{(n, q)}\right) u_{j}^{(n, q)}-g\left(u_{j}^{(n, q)}\right) u_{j}^{(n, q)}-m_{0}\left(u_{j}^{(n, q)}\right)^{2}\right) d x\right] \\
\leqslant & -\frac{q^{2}}{2} \varphi^{\prime}\left(q N\left(u^{(n, q)}\right)\right) N\left(u^{(n, q)}\right) N^{\prime}\left(u^{(n, q)}\right)\left[u^{(n, q)}\right] \\
& -\frac{q}{2} \varphi\left(q N\left(u^{(n, q)}\right)\right) N^{\prime}\left(u^{(n, q)}\right)\left[u^{(n, q)}\right] \\
& +\int_{\mathbb{R}^{2}}\left(g\left(u^{(n, q)}\right) u^{(n, q)}+m_{0}\left(u^{(n, q)}\right)^{2}\right) d x \\
= & \left\|u^{(n, q)}\right\|^{2} .
\end{aligned}
$$

So, $u_{j}^{(n, q)} \rightarrow u^{(n, q)}$ in $H^{1}\left(\mathbb{R}^{2}\right)$. Hence $\tilde{\mathcal{J}}_{q}\left(0, u^{(n, q)}\right)=b_{n}(q)$ and $\tilde{\mathcal{J}}_{q}^{\prime}\left(0, u^{(n, q)}\right)=$ 0 , completing the proof.

\subsection{Conclusion of the proof}

Now the proof of Theorem 1.1 can be concluded. Let $n \in \mathbb{N}^{*}$ and consider $b_{1}(q)<\ldots<b_{n}(q)$ and the corresponding critical points $u_{1}^{(n, q)}, \ldots, u_{n}^{(n, q)}$ of $\mathcal{J}_{q}$. In fact, we show that, for $q$ small enough, $u_{1}^{(n, q)}, \ldots, u_{n}^{(n, q)}$ are critical points of $J_{q}$ and so solutions of (1.1). Indeed, by Proposition 3.10 we know that there exists $C(n)>0$ such that, for every $i=1, \ldots, n,\left\|u_{i}^{(n, q)}\right\| \leqslant C(n)$. If by contradiction, there exists $i=1, \ldots, n$ such that $q N\left(u_{i}^{(n, q)}\right)>1$, we have

$$
\frac{1}{q}<N\left(u_{i}^{(n, q)}\right) \leqslant C\left\|u_{i}^{(n, q)}\right\|^{6} \leqslant C(n),
$$

but this is not possible for $q$ small.

\section{References}

[1] Azzollini, A., d'Avenia, P., Pomponio, A.: Multiple critical points for a class of nonlinear functionals. Ann. Mat. Pura Appl. (4) 190, 507-523 (2011)

[2] Berestycki, H., Gallouët, T., Kavian, O.: Équations de Champs scalaires euclidiens non linéaires dans le plan. C R. Acad. Sci. Paris Sér. I Math. 297, 307-310 (1983) and Publications du Laboratoire d'Analyse Numérique, Université de Paris VI (1984)

[3] Berestycki, H., Lions, P.-L.: Nonlinear scalar field equations. I. Existence of a ground state. Arch. Rational Mech. Anal. 82, 313-345 (1983)

[4] Berestycki, H., Lions, P.-L.: Nonlinear scalar field equations. II. Existence of infinitely many solutions. Arch. Rational Mech. Anal. 82, 347-375 (1983) 
[5] Berti, M., Bolle, P.: Periodic solutions of nonlinear wave equations with general nonlinearities. Commun. Math. Phys. 243, 315-328 (2003)

[6] Byeon, J., Huh, H., Seok, J.: Standing waves of nonlinear Schrödinger equations with the gauge field. J. Funct. Anal. 263, 1575-1608 (2012)

[7] Deser, S., Jackiw, R., Templeton, S.: Topologically massive gauge theories. Ann. Phys. 140, 372-411 (1982)

[8] Felsager, B.: Geometry, Particles, and Fields. Springer, New York (1998)

[9] Hagen, C.: A new gauge theory without an elementary photon. Ann. Phys. 157, 342-359 (1984)

[10] Hirata, J., Hikoma, N., Tanaka, K.: Nonlinear scalar field equations in $\mathbb{R}^{N}$ : mountain pass and symmetric mountain pass approaches. Topol. Methods Nonlinear Anal. 35, 253-276 (2010)

[11] Huh, H.: Standing waves of the Schrödinger equation coupled with the ChernSimons gauge field. J. Math. Phys. 53, 063702-063708 (2012)

[12] Jackiw, R., Pi, S.-Y.: Soliton solutions to the gauged nonlinear Schrödinger equation on the plane. Phys. Rev. Lett. 64, 2969-2972 (1990)

[13] Jackiw, R., Pi, S.-Y.: Classical and quantal nonrelativistic Chern-Simons theory. Phys. Rev. D (3) 42, 3500-3513 (1990)

[14] Jeanjean, L.: Existence of solutions with prescribed norm for semilinear elliptic equations. Nonlinear Anal. 28, 1633-1659 (1997)

[15] Jeanjean, L., Le Coz, S.: An existence and stability result for standing waves of nonlinear Schrödinger equations. Adv. Differ. Equ. 11, 813-840 (2006)

[16] Jeong, W., Seok, J.: On perturbation of a functional with the mountain pass geometry: applications to the nonlinear Schrödinger-Poisson equations and the nonlinear Klein-Gordon-Maxwell equations. Calc. Var. Partial Differ. Equ. 49, 649-668 (2014)

[17] Lerda, A.: Anyons: Quantum Mechanics of Particles with Fractional Statistics. Springer, Berlin, Hidelberg (1992)

[18] Naber, G.L.: Topology, Geometry, and Gauge Fields: FoundationsInteractions. Springer, New York (2011)

[19] Pomponio, A., Ruiz, D.: A Variational Analysis of a Gauged Nonlinear Schrödinger Equation. J. Eur. Math. Soc. (to appear)

[20] Pomponio, A., Ruiz, D.: Boundary concentration of a Gauged nonlinear Schrödinger equation on large balls. Calc. Var. Partial Differ. Equ. 53, 289$316(2015)$

[21] Schonfeld, J.F.: A mass term for three-dimensional gauge fields. Nucl. Phys. B 185, 157-171 (1981) 
[22] Tarantello, G.: Self-dual Gauge field Vortices: An Analytical Approach. Birkäuser, Boston (2007)

[23] Wan, Y., Tan, J.: Standing waves for the Chern-Simons-Schrödinger systems without (AR) condition. J. Math. Anal. Appl. 415, 422-434 (2014)

[24] Wilczek, F.: Fractional Statistics and Anyon Superconductivity. World Scientific, Teaneck (1990)

Patricia L. Cunha

Departamento de Informática e Métodos Quantitativos

Fundação Getulio Vargas

Av. Nove de Julho, 2029 - Bela Vista

São Paulo, SP, 01313-902

Brazil

e-mail: patcunha80@gmail.com

Pietro d'Avenia, Alessio Pomponio

Dipartimento di Meccanica, Matematica e Management

Politecnico di Bari

Via E. Orabona 4

70125 Bari

Italy

e-mail: pietro.davenia@poliba.it

Alessio Pomponio

e-mail: alessio.pomponio@poliba.it

Gaetano Siciliano

Departamento de Matemática

Universidade de São Paulo

Rua do Matão, 1010 - Cidade Universitaria

São Paulo, SP, 05508-090

Brazil

e-mail: sicilian@ime.usp.br

Received: 12 February 2015.

Accepted: 5 August 2015. 